\title{
OCORRÊNCIA DE OTITE CANINA POR Pseudomonas spp. E SENSIBILIDADE DOS ISOLADOS REGISTRADOS NO LABORATÓRIO REGIONAL DE DIAGNÓSTICO NO PERÍODO DE DEZ ANOS
} GONÇALVES, Helena Piúma ${ }^{2}$; CLEFF, Marlete Brum ${ }^{3}$; SILVA, Cristine Cioato da ${ }^{4}$; MATOS, Caroline Bohnen de ${ }^{4}$; PERERA, Soliane Carra ${ }^{4}$; GIORDANI, Claudia ${ }^{5}$; LADEIRA, Silvia Leal ${ }^{6}$.

${ }^{1}$ Médica Veterinária, Mestre, Professora, Faculdade de Medicina Veterinária/UCS; ${ }^{2}$ Médica Veterinária, Doutoranda, Programa de Pós-Graduação em Veterinária/UFPEL; ${ }^{3}$ Médica Veterinária, Doutora, Professora, Departamento de Clínicas Veterinárias/UFPEL; ${ }^{4}$ Médica Veterinária, Mestre, Programa de Pós-Graduação em Veterinária/UFPEL; ${ }^{5}$ Médica Veterinária, Doutora, Professora, Faculdade de Medicina Veterinária/UCS; ${ }^{6}$ Médica Veterinária, Doutora, Laboratório Regional de Diagnóstico, Faculdade de Veterinária/UFPEL.

\section{RESUMO}

A otite canina destaca-se na clínica veterinária, sendo que dentre os bacilos Gramnegativos, o gênero Pseudomonas apresenta-se como fator complicador no tratamento, caracterizando-se como um grande desafio para os clínicos, em virtude do perfil de multirresistência. Devido à importância clínica, verificaram-se os registros de casos de otite em cães causados pela bactéria, no período de 2009 a 2019, no Laboratório Regional de Diagnóstico (LRD) da Faculdade de Veterinária da Universidade Federal de Pelotas e o perfil de resistência dos isolados bacterianos. Assim, realizou-se o levantamento da casuística de otites registradas no LRD, sendo incluídas amostras de cães com queixa ou sinais clínicos de otite e selecionadas aquelas com isolamento de Pseudomonas spp. Como resultados, Pseudomonas spp. esteve presente em $11,7 \%$ dos casos, o que está de acordo com dados da literatura que cita uma frequência de 3,4 a 31,6\%. A resistência de Pseudomonas spp. aos fármacos beta-lactâmicos foi expressiva, com $87 \%$ de resistência à amoxicilina com clavulanato, cefalotina, cefalexina e oxacilina, seguido de $84,6 \%$ para ampicilina e $15,4 \%$ para ceftriaxona. A média de resistência para outros fármacos foi de $73,9 \%$ para fluorquinolonas e $52,2 \%$ para sulfonamidas e aminoglicosídeos. O estudo destaca a importância da avaliação microbiológica de amostras otológicas, especialmente quando Pseudomonas spp. estiver presente, já que o diagnóstico definitivo é fundamental para a implementação de terapia individualizada para os animais, assim como para o controle da resistência bacteriana.

Palavras-chave: Antibióticos. Bactérias. Casuística. Resistência. 


\section{INTRODUÇÃO}

O gênero Pseudomonas é frequentemente isolado de infecções cutâneas em cães e demonstra grande importância em casos de otite externa e média (ARAIS, 2013). Segundo a literatura, Pseudomonas spp. tem sido considerada a bactéria Gram-negativa de maior prevalência nas otites caninas, caracterizando-as como uma das infecções otológicas mais desafiadoras em relação à terapia, devido à resistência intrínseca aos antimicrobianos e fácil multiplicação da bactéria no conduto auditivo alterado por inflamações crônicas (NETO; LOPES, 2016; OLIVEIRA et al., 2012).

Desde a introdução dos primeiros antibacterianos até os dias atuais, vem sendo registrado um aumento da população de microrganismos resistentes, causado, entre outros motivos, pela pressão de seleção exercida através do uso inadequado e indiscriminado desses fármacos. Acresce que a multirresistência constitui uma ameaça à saúde pública, somada à incapacidade da indústria farmacêutica em disponibilizar respostas terapêuticas para alguns gêneros bacterianos (COLARES et al., 2016; FERRAREZE et al., 2007). Devido ao uso abusivo de antimicrobianos e outros fatores associados à terapêutica das otites em cães, a incidência de multirresistência em Pseudomonas spp. é crescente (SILVA et al., 2016). Também, Pseudomonas spp. destaca-se como um dos microrganismos Gram-negativos mais frequentemente envolvidos em infecções hospitalares em humanos (COSTA, 2009), e o tratamento clínico de infecções causadas pelo gênero torna-se um desafio para as equipes de saúde de modo geral, visto o caráter de transmissão interespécies (FERRAREZE et al., 2007). Ainda, a resistência a múltiplas drogas tem sido observada em isolados de cães, com uma taxa de $34,9 \%$ de resistência do gênero Pseudomonas a três ou mais fármacos e, de 15,5\% a cinco ou mais dos antimicrobianos avaliados (OLIVEIRA et al., 2012). Assim sendo, dada a importância desta bactéria em saúde humana e veterinária, objetivou-se verificar os registros de casos de otite canina causados por Pseudomonas spp. no período de dez anos no Laboratório Regional de Diagnóstico (LRD) da Faculdade de Veterinária da Universidade Federal de Pelotas (FaVet/UFPEL), bem como avaliar o perfil de sensibilidade dos isolados registrados aos antimicrobianos, enfatizando a necessidade da realização dos testes de identificação e susceptibilidade, minimizando os casos de resistência. 


\section{MATERIAL E MÉTODOS}

Com o interesse de avaliar a ocorrência do gênero Pseudomonas em amostras de otite canina encaminhadas para diagnóstico, foi realizado um levantamento nos laudos de exames do LRD da FaVet/UFPEL, através da análise retrospectiva dos laudos emitidos durante o período de dez anos, sendo incluídos dados de agosto de 2009 até agosto de 2019. Foram digitalizados os dados referentes a animais atendidos em clínicas veterinárias da cidade de Pelotas e da região sul do Rio Grande do Sul e do Hospital de Clínicas Veterinárias da FaVet/UFPEL. Os critérios utilizados para inclusão dos dados foram o recebimento de amostras de secreção otológica de cães de ambos os sexos, com queixa ou sinais clínicos de otite, encaminhadas para cultura bacteriana e/ou antibiograma. Foram selecionadas a partir destes, os casos de otite por Pseudomonas spp. Os dados referentes a outras espécies bacterianas foram excluídos ou desconsiderados, além dos fatores como tempo de tratamento anterior, medicações prévias ou em andamento, assim como raça e idade. Ainda, foram compilados os dados dos isolados de Pseudomonas spp. avaliados quanto à sensibilidade/resistência através da medição $(\mathrm{mm})$ dos halos de inibição de crescimento segundo Bauer (1966), solicitados pelos clínicos veterinários no período do estudo. Nos antibiogramas, foram utilizados os seguintes fármacos: ampicilina (10 mcg), amoxicilina com clavulanato $(20 / 10 \mathrm{mcg}$ ), amicacina $(30 \mathrm{mcg})$, cefalexina (30 mcg), ceftriaxona (30 mcg), cefalotina (30 mcg) enrofloxacina $(5 \mathrm{mcg}$ ), ciprofloxacina $(5 \mathrm{mcg})$, neomicina (30 mcg), gentamicina (10 mcg), oxacilina (1 mcg), imipenem (10 mcg) e sulfametoxazol associado com trimetoprim ( $25 \mathrm{mcg})$.

\section{RESULTADOS E DISCUSSÃO}

A partir da análise das fichas do LRD, foram encontrados 4.720 laudos de cães emitidos no período de dez anos, destes, 197 laudos microbiológicos corresponderam a casos de enfermidade otológica, ocasionadas por agentes diversos. No período avaliado, 23 cães apresentaram Pseudomonas spp., como agente etiológico de otite, correspondendo a 11,7\% (23/197) do total de amostras (Tabela 1$)$. 
Tabela 1 - Isolamento de Pseudomonas spp. das amostras de otite em cães, recebidas pelo Laboratório Regional de Diagnóstico da Universidade Federal de Pelotas, no período de agosto de 2009 a agosto de 2019.

\begin{tabular}{cccc}
\hline ANO & CASOS DE OTITE & Pseudomonas spp. (n) & Pseudomonas spp. (\%) \\
\hline 2009 & 2 & 0 & 0 \\
2010 & 5 & 0 & 0 \\
2011 & 21 & 4 & 19 \\
2012 & 10 & 0 & 0 \\
2013 & 5 & 2 & 40 \\
2014 & 5 & 0 & 0 \\
2015 & 25 & 2 & 8 \\
2016 & 23 & 3 & 13 \\
2017 & 49 & 4 & 8,2 \\
2018 & 30 & 5 & 16,7 \\
2019 & 22 & 3 & 13,6 \\
\hline TOTAL & 197 & 23 & 11,7 \\
\hline
\end{tabular}

Ao analisarmos os dados do LRD referentes aos diagnósticos de otite microbiana em cães ( $n=197$ ) observamos uma média de 19,7 casos/ano. Os resultados encontrados em relação à ocorrência de otite são inferiores à casuística obtida em outros estudos, já que a otite externa canina representa um percentual expressivo dos casos atendidos na prática clínica veterinária no Brasil (GHELLER et al., 2017; OLIVEIRA et al., 2012). Conforme nossa rotina, dados de relatórios de estágio e residência e atendimentos no ambulatório veterinário (MORAES et al., 2014), demonstraram que a otite canina está entre as principais dermatopatias em cães, assim como observado por diversos autores (ARAIS, 2013; GHELLER et al., 2017; SILVA et al., 2014). A divergência entre os dados do nosso estudo e a casuística clínica relatada na literatura, pode ser explicada devido ao não encaminhamento de amostras pelos clínicos na região, que optam por tratar empiricamente os pacientes, buscando um diagnóstico de certeza apenas quando não há resposta eficaz frente a terapêutica instituída, prejudicando desta forma a resolução da enfermidade e o registro de dados, o que também está de acordo com outros autores (ARAIS, 2013).

Dentre os agentes etiológicos observados nas amostras de otite encaminhadas ao LRD, foram observadas bactérias Gram-positivas, evidenciando-se principalmente o gênero Staphylococcus, e bactérias Gram-negativas destacando-se os gêneros Proteus e Pseudomonas, concordando com a literatura, com ênfase para a espécie Pseudomonas aeruginosa nos quadros de otite em cães (GHELLER et al., 2017; MARTINS et al., 2011). 
Dados da literatura relatam que o isolamento de $P$. aeruginosa ocorre entre 3,4 a $31,6 \%$ das amostras clínicas de cães com otite externa (ARAIS, 2013), sendo que Oliveira et al. (2005), ao avaliarem 433 cepas isoladas de otite externa em cães, observaram 25\% isolados de $P$. aeruginosa, enquanto que Neto e Lopes (2016), ao avaliarem 187 amostras por cultura bacteriana, observaram 47\% de predominância de bactérias Gram-negativas e 16\% foram identificadas como P. aeruginosa. Entretanto, Martins et al. (2011), ao avaliarem o prontuário de 274 cães com otite, atendidos em hospital veterinário, observaram uma ocorrência de $12,89 \%$ de Pseudomonas spp., resultado próximo aos $11,7 \%$ de isolamento observados em nosso estudo.

É notória a emergência de resistência de Pseudomonas spp. aos fármacos antimicrobianos presentes nos produtos otológicos (ARAIS, 2013), sendo que $P$. aeruginosa tem demonstrado resistência a vários antibióticos amplamente utilizados na medicina veterinária (ARAIS, 2013; TUNON et al., 2008). A resistência aos antimicrobianos nos casos de otite está relacionada à pressão seletiva exercida pelo uso intenso e indiscriminado de medicamentos, assim como a fatores intrínsecos relacionados ao conduto auditivo dos pacientes caninos e às bactérias, trazendo preocupação para a área médica em geral (FERREIRA, 2005).

Os isolados de Pseudomonas spp. provenientes do LRD no período estudado e avaliados quanto à sensibilidade aos antimicrobianos demonstraram multirresistência a fármacos. Neto e Lopes (2016), ao avaliarem amostras de otites em cães, isolaram diferentes bactérias com perfil de multirresistência, sendo o maior índice observado no gênero Pseudomonas, com resistência a três ou mais classes de antibióticos, o que está de acordo com os resultados observados no presente estudo. Ao avaliar os grupos de antibióticos, observou-se alto índice de resistência de Pseudomonas spp. aos fármacos beta-lactâmicos, com $87 \%$ de resistência frente à amoxicilina com clavulanato, cefalotina, cefalexina e oxacilina, seguido de $84,6 \%$ para ampicilina e 15,4\% para ceftriaxona. Para outros grupos de fármacos, a média de resistência foi de 52,2\% para sulfonamidas e aminoglicosídeos com 15,4\% para amicacina e 7,7\% para neomicina, enquanto para fluorquinolonas o percentual foi de $73,9 \%$ (Tabela 2 ). 
Tabela 2 - Isolados clínicos de Pseudomonas spp. e susceptibilidade frente aos diferentes grupos de antimicrobianos testados.

\begin{tabular}{|c|c|c|c|c|}
\hline Isolado & Betalactâmicos & Aminoglicosídeos & Fluorquinolonas & Sulfonamidas \\
\hline 1 & $2(\mathrm{R}) 0(\mathrm{~S})$ & $1(\mathrm{R}) 0(\mathrm{~S})$ & $1(\mathrm{R}) 0(\mathrm{~S})$ & $1(R) 0(S)$ \\
\hline 2 & $2(\mathrm{R}) 0(\mathrm{~S})$ & $0(\mathrm{R}) 2(\mathrm{~S})$ & $1(\mathrm{R}) 0(\mathrm{~S})$ & $1(\mathrm{R}) 0(\mathrm{~S})$ \\
\hline 3 & $1(\mathrm{R}) 0(\mathrm{~S})$ & $0(\mathrm{R}) 1(\mathrm{~S})$ & $0(\mathrm{R}) 2(\mathrm{~S})$ & $0(\mathrm{R}) 0(\mathrm{~S})$ \\
\hline 4 & $2(\mathrm{R}) 0(\mathrm{~S})$ & $0(\mathrm{R}) 1(\mathrm{~S})$ & $1(\mathrm{R}) 1(\mathrm{~S})$ & $1(\mathrm{R}) 0(\mathrm{~S})$ \\
\hline 5 & $3(\mathrm{R}) 0(\mathrm{~S})$ & $2(R) 1(S)$ & $0(\mathrm{R}) 0(\mathrm{~S})$ & $0(R) 0(S)$ \\
\hline 6 & $0(\mathrm{R}) 1(\mathrm{~S})$ & $0(R) 2(S)$ & $0(\mathrm{R}) 2(\mathrm{~S})$ & $0(R) 0(S)$ \\
\hline 7 & $1(\mathrm{R}) 0(\mathrm{~S})$ & $2(\mathrm{R}) 1(\mathrm{~S})$ & $1(\mathrm{R}) 0(\mathrm{~S})$ & $0(\mathrm{R}) 0(\mathrm{~S})$ \\
\hline 8 & $1(\mathrm{R}) 0(\mathrm{~S})$ & $1(R) 1(S)$ & $2(\mathrm{R}) 0(\mathrm{~S})$ & $1(\mathrm{R}) 0(\mathrm{~S})$ \\
\hline 9 & $4(\mathrm{R}) 0(\mathrm{~S})$ & $0(\mathrm{R}) 3(\mathrm{~S})$ & $1(\mathrm{R}) 0(\mathrm{~S})$ & $1(R) 0(S)$ \\
\hline 10 & $2(\mathrm{R}) 0(\mathrm{~S})$ & $1(\mathrm{R}) 0(\mathrm{~S})$ & $1(\mathrm{R}) 0(\mathrm{~S})$ & $1(R) 0(S)$ \\
\hline 11 & $1(\mathrm{R}) 0(\mathrm{~S})$ & $0(\mathrm{R}) 2(\mathrm{~S})$ & $1(\mathrm{R}) 1(\mathrm{~S})$ & $1(\mathrm{R}) 0(\mathrm{~S})$ \\
\hline 12 & $2(\mathrm{R}) 2(\mathrm{~S})$ & $0(\mathrm{R}) 2(\mathrm{~S})$ & $2(\mathrm{R}) 0(\mathrm{~S})$ & $1(R) 0(S)$ \\
\hline 13 & $3(R) 0(S)$ & $0(R) 2(S)$ & $0(\mathrm{R}) 1(\mathrm{~S})$ & $1(R) 0(S)$ \\
\hline 14 & $1(\mathrm{R}) 1(\mathrm{~S})$ & $0(\mathrm{R}) 2(\mathrm{~S})$ & $0(\mathrm{R}) 2(\mathrm{~S})$ & $0(\mathrm{R}) 0(\mathrm{~S})$ \\
\hline 15 & $1(\mathrm{R}) 1(\mathrm{~S})$ & $2(R) 0(S)$ & $1(\mathrm{R}) 1(\mathrm{~S})$ & $0(\mathrm{R}) 0(\mathrm{~S})$ \\
\hline 16 & $2(\mathrm{R}) 1(\mathrm{~S})$ & $0(\mathrm{R}) 2(\mathrm{~S})$ & $0(\mathrm{R}) 2(\mathrm{~S})$ & $0(R) 0(S)$ \\
\hline 17 & $0(\mathrm{R}) 3(\mathrm{~S})$ & $0(\mathrm{R}) 2(\mathrm{~S})$ & $0(\mathrm{R}) 2(\mathrm{~S})$ & $1(R) 0(S)$ \\
\hline 18 & $4(\mathrm{R}) 0(\mathrm{~S})$ & $0(\mathrm{R}) 0(\mathrm{~S})$ & $0(\mathrm{R}) 2(\mathrm{~S})$ & $1(\mathrm{R}) 0(\mathrm{~S})$ \\
\hline 19 & $2(\mathrm{R}) 0(\mathrm{~S})$ & $0(\mathrm{R}) 2(\mathrm{~S})$ & $1(\mathrm{R}) 1(\mathrm{~S})$ & $0(\mathrm{R}) 0(\mathrm{~S})$ \\
\hline 20 & $2(\mathrm{R}) 0(\mathrm{~S})$ & $0(\mathrm{R}) 1(\mathrm{~S})$ & $1(\mathrm{R}) 1(\mathrm{~S})$ & $0(R) 0(S)$ \\
\hline 21 & $0(\mathrm{R}) 0(\mathrm{~S})$ & $1(\mathrm{R}) 2(\mathrm{~S})$ & $1(\mathrm{R}) 1(\mathrm{~S})$ & $0(\mathrm{R}) 0(\mathrm{~S})$ \\
\hline 22 & $2(\mathrm{R}) 0(\mathrm{~S})$ & $1(\mathrm{R}) 1(\mathrm{~S})$ & $1(\mathrm{R}) 1(\mathrm{~S})$ & $1(\mathrm{R}) 0(\mathrm{~S})$ \\
\hline 23 & $2(\mathrm{R}) 0(\mathrm{~S})$ & $1(\mathrm{R}) 1(\mathrm{~S})$ & $1(\mathrm{R}) 1(\mathrm{~S})$ & $0(\mathrm{R}) 0(\mathrm{~S})$ \\
\hline
\end{tabular}

${ }^{*} \mathrm{R}=$ resistente; $\mathrm{S}=$ sensível. 
Os índices de resistência de Pseudomonas spp. aos antimicrobianos observados neste estudo foram elevados, e reafirmam os relatos de outros autores. Souza et al. (2006) descreveram a multirresistência em 135 linhagens de Pseudomonas isoladas de otite em cães. Oliveira et al. (2012) argumentam que os casos de otite podem ser facilitados pela veiculação hídrica de Pseudomonas para o conduto auditivo dos animais, o que poderia perpetuar a presença da bactéria. Ainda, Pseudomonas tem sido comumente isolada de cães e gatos com piodermites, infecções urinárias, infecções oculares, osteomielites, dentre outras afecções (ARAIS, 2013). Em unidades de terapia intensiva de humanos é citada, frequentemente, a transmissão de isolados multirresistentes entre os pacientes, constituindo um grave problema de saúde pública devido às características do gênero, já que Pseudomonas spp. produzem várias toxinas e enzimas que aumentam sua virulência frente ao hospedeiro (FERREIRA, 2005; MATA; ABEGG, 2007; SILVA et al., 2016).

Entre os fatores de patogenicidade, citam-se: a secreção de polissacarídeos extracelulares, que interferem na fagocitose, prejudicando a difusão de antibióticos; produção de toxina $A$ por $90 \%$ das cepas, uma proteína extracelular envolvida na patogênese da bactéria; além da exoenzima-S, que interfere na síntese proteica e ocasiona lesão tecidual. A produção de proteases por Pseudomonas pode contribuir para a ulceração do canal auditivo e a perfuração da membrana timpânica, explicando assim a ocorrência mais comum desta complicação em cães com otite (SARIDOMICHELAKIS et al., 2007). Ainda, algumas cepas produzem pigmentos, como a piocianina, que retarda o crescimento de outras bactérias, facilitando sua proliferação (MATA; ABEGG, 2007). A produção de enzimas que alteram o tecido conjuntivo, como as elastases (LasA e LasB), que degradam elastina, e uma protease alcalina (AprA) que degrada o colágeno, também têm sido relacionadas à patogenicidade do gênero Pseudomonas (FERREIRA, 2005).

Devido a estes diversos fatores, os médicos veterinários devem estar atentos às falhas terapêuticas ocasionadas pelo uso empírico de antimicrobianos (CARDINAL et al., 2015; COLARES et al., 2016). Sendo que o conhecimento da etiologia e o perfil de susceptibilidade das bactérias têm grande importância, principalmente quando houver envolvimento de 
Pseudomonas spp. nas infecções, para assim estabelecer um tratamento específico e eficaz, além de evitar a disseminação de bactérias multirresistentes (TUNON et al., 2008).

\title{
CONCLUSÃO
}

Pseudomonas spp. esteve presente em amostras de otite canina diagnosticadas no LRD da FaVet/UFPEL no período estudado, sendo observados elevados índices de multirresistência, destacando-se os fármacos beta-lactâmicos, fluorquinolonas, sulfonamidas e aminoglicosídeos. Estudos relacionados aos agentes de otites em cães são importantes, já que este é um problema frequente na rotina clínica, e que vem sendo tratado, muitas vezes, de forma empírica e sem a devida avaliação microbiológica das amostras, o que é fundamental para a implementação de terapia individualizada para os animais, assim como para o controle da resistência bacteriana.

\section{OCCURRENCE OF CANINE OTITIS BY Pseudomonas spp. AND SENSITIVITY OF THE ISOLATES REGISTERED IN THE REGIONAL DIAGNOSTIC LABORATORY IN A TEN YEAR PERIOD}

ABSTRACT

\begin{abstract}
C anine otitis stands out in veterinary clinics, and among the Gram-negative bacilli, the genus Pseudomonas presents a complicating agent for the treatment, being a major challenge for clinicians due to its multidrug resistance profile. Due to the clinical importance, the case records of canine otitis caused by the bacterium and the resistance profile of the bacteria from 2009 to 2019 were verified, at the Regional Diagnostic Laboratory (RDL) of the Veterinary Faculty from the Federal University of Pelotas. The survey of cases of otitis registered in RDL was performed, including dog samples with complaints or clinical signs of otitis, and those with isolation of Pseudomonas spp. were selected. As results, Pseudomonas spp. was present in $11.7 \%$ of cases, what is in agreement with the literature data, which cites a frequency of $3.4 \%$ to $31.6 \%$. The resistance of Pseudomonas spp. for beta-lactam antibiotics was expressive, with $87 \%$ of resistance to amoxicillin with clavulanate, cephalothin, cephalexin and oxacillin, followed by $84.6 \%$ for ampicillin and $15.4 \%$ for ceftriaxone. The average resistance for other drugs was $73.9 \%$ for fluoroquinolones and $52.2 \%$ for sulfonamides and aminoglycosides. The study highlights the importance of microbiological evaluation of otological samples, especially when Pseudomonas spp. is present, since the definitive diagnosis is fundamental for the
\end{abstract}


implementation of individualized therapy for the animal, as well as for the control of bacterial resistance.

Keywords: Antibiotics. Bacteria. Casuistry. Resistance.

\section{OCURRENCIA DE OTITIS CANINA POR Pseudomonas spp. Y SENSIBILIDAD DE LOS AISLAMIENTOS REGISTRADOS EN EL LABORATORIO REGIONAL DE DIAGNOSTICO EN UN PERÍODO DE DIEZ AÑOS}

\section{RESUMEN}

a otitis canina se destaca en la clínica veterinaria, y entre los bacilos Gram-negativos, el género Pseudomonas se presenta como un agente complicado del tratamiento, siendo un desafío importante para los clínicos por el perfil de resistencia a múltiples drogas. Debido a la importancia clínica, se estudiaron los casos de otitis canina causados por la bacteria entre 2009 y 2019, en el Laboratorio Regional de Diagnostico (LRD) de la Facultad de Veterinaria de Universidad Federal de Pelotas y el perfil de resistencia de los aislamientos bacterianos. Así, se realizó el levantamiento de casos de otitis registrados en el LRD, incluidas muestras de perros con signos clínicos de otitis y seleccionando aquellos con aislamiento de Pseudomonas spp. Como resultado, Pseudomonas spp. estuvo presente en el $11,7 \%$ de los casos, lo que está de acuerdo con los datos de la literatura, que cita una frecuencia del 3,4\% al 31,6\%. La resistencia de Pseudomonas spp. a los fármacos betalactámicos fue expresiva, con un $87 \%$ de resistencia a la amoxicilina con clavulanato, cefalotina, cefalexina y oxacilina, seguidos de $84,6 \%$ para ampicilina y $15,4 \%$ para ceftriaxona. La resistencia promedio para otras drogas fue del $73,9 \%$ para las fluorquinolonas y del $52,2 \%$ para las sulfonamidas y aminoglucósidos. El estudio destaca la importancia de la evaluación microbiológica de muestras otológicas, especialmente cuando Pseudomonas spp. es presente, ya que el diagnóstico definitivo es fundamental para la implementación de la terapia individualizada para cada animal, así como para el control de la resistencia bacteriana.

Palabras clave: Antibióticos. Bacterias. Casuística. Resistencia.

\section{REFERÊNCIAS}

ARAIS, L. R. Resistência aos antimicrobianos, pesquisa de exotoxinas e relação genética de Pseudomonas aeruginosa isoladas de otite externa e piodermite canina. Niterói: UFF, 2013. 105p. Tese (Doutorado em Clínica e Reprodução Animal), Faculdade de Medicina Veterinária, Universidade Federal Fluminense, Niterói, 2013. 
BAUER, A. W. Kirby Bauer method antimicrobial susceptibility testing by a standardized single disk method. American Journal of Clinical Pathology, v. 45, n. 4, p. 493-496, 1966.

CARDINAL, L. D. S. M.; CRUZ, F. A. F. D.; MENESES, J. P. D.; et al. Análise da terapia antimicrobiana empírica em infecção de corrente sanguínea por Pseudomonas aeruginosa em um hospital universitário. Revista da Sociedade Brasileira de Clínica Médica, v. 13, n. 4, p. 257-261, 2015.

COLARES, K. T. P.; ANDRADE, A. F.; ATHAYDE, L. A. Ocorrência de Pseudomonas aeruginosa multirresistente em unidade de tratamento intensivo: uma revisão. Revista Brasileira de Análises Clínicas - Suplemento Especial de Microbiologia e Micologia, v. 48, n. 3, supl. 1, p. 15-19, 2016.

COSTA, A. C. Atividade antibacteriana dos óleos essenciais de $\boldsymbol{O}$. vulgare $L$. e Cinnamonum zeylanicum B. contra bactérias multirresistentes. Paraíba: UFPB, 2009. 98p. Tese (Doutorado em Produtos Naturais e Sintéticos Bioativos), Faculdade de Farmácia, Universidade Federal da Paraíba, 2009.

FERRAREZE, M. V. G.; LEOPOLDO, V. C.; ANDRADE, D.; et al. Pseudomonas aeruginosa multirresistente em unidade de cuidados intensivos: desafios que procedem? Acta Paulista de Enfermagem, v. 20, n. 1, p. 7-11, 2007.

FERREIRA, L. L. Estrutura clonal e multirresistência em Pseudomonas aeruginosa. Rio de Janeiro: FIOCRUZ, 2005. 114 p. Dissertação (Mestrado em Vigilância Sanitária), Programa de Pós-Graduação em Vigilância Sanitária, Fundação Oswaldo Cruz, 2005.

GHELLER, B. G.; MEIRELLES, A. C. F.; FIGUEIRA, P. T.; et al. Patógenos bacterianos em cães com otite externa e seus perfis de suscetibilidade a diversos antimicrobianos. Pubvet, v. 11, n. 2, p. 159-167, 2017.

MARTINS, E. A.; MOMESSO, C. S.; NARDO, C. D. D.; et al. Estudo clínico e microbiológico de otite externa de cães atendidos em hospital veterinário do noroeste paulista. Acta Veterinaria Brasilica, v. 5, n. 1, p. 61-67, 2011.

MATA, P. T. G.; ABEGG, M. A. Descrição de caso de resistência a antibióticos por Pseudomonas aeruginosa. Arquivos do Mudi, v. 11, n. 2, p. 20-25, 2007.

MORAES, T. B.; AZAMBUJA, R. H. M.; AÑANÃ, D. C.; et al. Casuística de doenças tegumentares no Ambulatório CEVAL-UFPel. In: CONGRESSO BRASILEIRO DE MEDICINA VETERINÁRIA, 41, 2014, Gramado. ANAIS. Gramado: SOVERGS, 2014. Disponível em: <http://www.sovergs.com.br/BKP/site_/conbravet2014/artigos/trabalhos_2044.htm>.

NETO, S. A.; LOPES, C. M. Estudo retrospectivo da ocorrência, sensibilidade e resistência antimicrobiana in vitro em otopatias caninas na região da grande Florianópolis - SC. Revista 
de Educação Continuada em Medicina Veterinária e Zootecnia do CRMV-SP, v. 13, n. 3, p. 37, 2016.

OLIVEIRA, L. C.; MEDEIROS, C. M. O.; SILVA, I. N. G.; MONTEIRO, A. J.; LEITE, C. A. L.; CARVALHO, C. B. M. Susceptibilidade a antimicrobianos de bactérias isoladas de otite externa em cães. Arquivo Brasileiro de Medicina Veterinária e Zootecnia, v. 57, n. 3, p. 405408, 2005.

OLIVEIRA, V. B.; RIBEIRO, M. G.; ALMEIDA, A. C. S.; et al. Etiologia, perfil de sensibilidade aos antimicrobianos e aspectos epidemiológicos na otite canina: estudo retrospectivo de 616 casos. Semina: Ciências Agrárias, v. 33, n. 6, p. 2367-2374, 2012.

SARIDOMICHELAKIS, M. N.; FARMAKI, R.; LEONTIDES, L. S.; et al. Aetiology of canine otitis externa: a retrospective study of 100 cases. Veterinary Dermatology, v. 18, n. 5, p. 341-347, 2007.

SILVA, A. P. D.; SCHMIDT, C.; VARGAS, A. C. D.; et al. Suscetibilidade antimicrobiana de Staphylococcus spp. isolados de cães com pioderma superficial. Pesquisa Veterinária Brasileira, v. 34, n. 4, p. 355-361, 2014.

SILVA, L. C. A.; PESSOA, D. A. N.; MAIA, L. A.; et al. Systemic Infection by Pseudomonas aeruginosa in a Dog. Acta Scientiae Veterinariae, v. 44, supl. 1, p. 1-5, 2016.

SOUZA, A. V. G.; SALERNO, T.; SIQUEIRA, A. K.; et al. Perfil de sensibilidade microbiana em 135 linhagens de Pseudomonas aeruginosa isoladas de cães com otite. In: REUNIÃO ANUAL DO INSTITUTO BIOLÓGICO DE SÃO PAULO, 19, 2006, São Paulo. ANAIS. São Paulo: IBSP, 2006.

TUNON, G. I. L.; SILVA, E. P.; FAIERSTEIN, C. C. Isolamento de estafilococos multirresistentes de otites em cães e sua importância para a saúde pública. BEPA - Boletim Epidemiológico Paulista, v. 5, n. 58, p. 04-07, 2008. 